\section{International Journal
and Food Technology}

Research Article

\title{
Vernonia amygdalina (BITTER LEAF) EXTRACTS AS PRESERVATIVE FOR CATFISH (Clarias gariepinus)
}

${ }^{1}$ R.N. Oladosu-Ajayi, ${ }^{2}$ H.E. Dienye, ${ }^{1}$ C.T. Ajayi and ${ }^{1}$ I.U. Agha

${ }^{1}$ Department of Fisheries Technology, FCFFT, New Bussa. Niger State

2 Department of Fisheries. University of Portharcourt, Rivers State

*Corresponding Author: H.E. Dienye, Department of Fisheries Technology, FCFFT, New Bussa. Niger State, E-mail: henrydienye@ gmail.com

Citation: H.E. Dienye, R.N. Oladosu-Ajayi, C.T. Ajayi and I.U. Agha (2016). Vernonia amygdalina (BITTER LEAF) EXTRACTS AS PRESERVATIVE FOR CATFISH (Clarias gariepinus) Int J Nutr Sci \& Food Tech 3:1, 102-108

Copyright: (C) 2017 H.E. Dienye et al. This is an open-access article distributed under the terms of the Creative Commons Attribution License, which permits unrestricted use, distribution, and reproduction in any medium, provided the original author and source are credited.

Received January 4, 2016; Accepted January 22, 2016; Published January 30, 2016.

\section{Abstract}

Ethanol, hot water and cold water extracts of bitter leaf (Vernonia amygdalina) were used as a preservative for catfish (Clarias gariepinus) to determine how far it can extend its shelf life. It was observed that the Ethanolic extract of bitter leaf was able to extend the shelf life of catfish (Clarias gariepinus) from the 24 hours as seen in the unpreserved sample to 36 hours post-slaughter after which it become unfit for consumption. The hot and cold water extracts could only preserved the fish for 20 hours post-slaughter. These two extract cannot thus be used to preserved catfish (Clarias gariepinus) as it could not even keep the fish fit for consumption for as long as it would have when unpreserved. Ethanol proved the best solvent of extracting bitter leaf because of its ability to liberate its phytochemical compounds unlike the hot and cold water extracts. This observation thus makes ethanolic extract of bitter leaf (Vernonia amygdalina) a useful natural fish preservative.

\section{Keywords: Vernonia amygdalina (Bitter leaf), Preservative, Extract, Catfish}

\section{Introduction}

Fish is a highly perishable commodity which spoils immediately after capture. The rate at which bacteria grow in fish is significantly reduced at low temperature. One of the simplest ways of lowering fish temperature thereby retarding bacteria growth is to ice the fish. As soon as the fish is iced, heat is transferred from the fish to the ice causing the ice to melt. This continues until an equilibrium temperature is reduced between the ice and the fish. This temperature will be a little above $\mathrm{O}^{0} \mathrm{C}$ if sufficient quantity of ice is used. At this temperature, the activities of micro organisms are reduced and the storage life of fish is prolonged. As the ice melts, the flowing water washes away bacteria and other debris from the fish. There have been many research studies regarding the shelf-life of fish stored in ice. Based on these studies, it is generally accepted that some tropical fish species can keep for longer periods in comparison to fish from temperate or colder water (Clucas, 1985). This can be attributed to differences in the bacteria growth rates in tropical fish stored in ice. However due to differences in the criteria used to define the limit of shelf-life, and methodologies used, comparison between shelf-life of fish from tropical and temperature waters is still difficult (Clucas, 1985). The herb known as the bitter leaf is a shrub or small tree that can reach twenty three feet in height when fully grown. Bitter leaf has a grey or brown coloured bark; the bark has a rough texture and is flaked. (Butter and Bailey, 1973)The leaves are used for human consumption and washed before eating to get rid of the bitter taste. They are used as vegetable and stimulate the digestive system, as well as they reduce fever. Furthermore, Vernonia amygdalina is also used instead of hops to make beer in Nigeria (Butter and Bailey, 1973). It has been observed to be eaten by goats in central zone of Delta state, Nigeria. However, in general has there been found, that Vernonia amygdalina have an astringent taste, which affects its intake (Bensi et al; 1995). The bitter taste is due to anti-nutritional factors such as alkaloids, saponins, tannins and glycosides (Butter and Bailey, 1973). This work is therefore undertaken to preserve and extend the shelf-life of freshly slaughtered catfish (Clarias gariepinus) using extracts of bitter leaf also determine which 
extract of Vernonia amygdalina has the best antimicrobial ability to stall spoilage in freshly slaughtered Clarias gariepinus.

\section{Materials and Methods}

\section{PREPARATION OF FISH SAMPLE}

The fish sample catfish (Clarias gariepinus) was purchased from Monday market in Kainji, Niger State. The fish was transported in kegs to the fish museum at Federal College of Freshwater Fisheries Technology, New Bussa, Niger State for the shelf-life studies. The fish used for the experiment was gutted before they were dipped in the three extracts separately. The working table was washed and later cleaned using ethanol.

\section{COLLECTION OF PLANT MATERIALS}

The plant materials used was bitter leaf (Vernonia amygdalina) and it was collected from Monday market in kanji, Niger State.

\section{PREPARATION OF BITTER LEAF EXTRACTS}

The bitter leaf was washed with clean water to remove the dust and dirt. This was then macerated and extraction done as follows:

$1.300 \mathrm{~g}$ of bitter leaf was soaked in $150 \mathrm{mls}$ of cold water for $24 \mathrm{hrs}$. The pulp obtained was left in clean, sterile glass container and shaken vigorously to allow for proper extraction. Filtration was done using a sterile muslin cloth after which the extract obtained, was air dried and stored at ambient temperature until it was used (Azu and Onyeagba, 2007)

2. $300 \mathrm{~g}$ of bitter leaf was soaked in $150 \mathrm{mls}$ of hot water for $24 \mathrm{hrs}$, and the resultant juice extraction was air dried and stored as done in (1) above.

3. $300 \mathrm{~g}$ of dry bitter leaf soaked in $150 \mathrm{mls}$ of $95 \%$ ethanol for $24 \mathrm{hrs}$ the resultant juice extracted was air dried and stored as done in (1) above.

\section{PRESERVATION OF FISH USING EXTRACTS}

The fish were dipped in the bitter leaf extracts in separate bowls.

\section{SHELF-LIFE STUDIES}

The fish samples that had been preserved in the extracts were monitored for spoilage at an interval of 4 hours and the organoleptic characteristics noted

\section{Results}

The results of this work showed a variation between catfish (Clarias gariepinus) samples preserved with cold water, hot water and ethanolic extracts of bitter leaf and the unpreserved sample. Table 1 shows the organoleptic changes of unpreserved catfish (Clarias gariepinus). This result showed that unpreserved catfish can stay for 24hours post-slaughter before spoilage can begin. Table 2 showed that catfish (Clarias gariepinus) preserved with ethanolic extract of bitter leaf (Vernonia amygdalina) stayed till the 36th hour post-slaughter before the spoilage characteristics could manifest. Table 3 showed the organoleptic characteristics of catfish (Clarias gariepinus) preserved with hot water extract of bitter leaf (Vernonia amygdalina) which stayed for $20 \mathrm{hrs}$ postslaughter before spoilage began. Table 4 showed that cold water extract of bitter leaf (Vernonia amygdalina) preserved catfish (Clarias gariepinus) for $20 \mathrm{hrs}$ post-slaughter before the onset of the spoilage.

\section{Discussion}

Food quality and safety have been concerns of mankind since the dawn of history, and in recent years there has been increasing disquiet on the part of governments, food processors and consumers (WHO,1995). Fish is a highly perishable commodity, more than cattle, sheep and poultry, and it gets spoiled very quickly after capture. Therefore, unless fish is disposed of quickly after capture, it must be preserved in some way (Ikeme and Bhandary, 2001). Fish preservation is an important method of extending the shelflife of fish in order to improve the quality of the products, for a longer period of time. The result of this work showed that catfish [Clarias gariepinus] can be preserved using natural preservatives [plant] which will extend its shelf-life and reduce post harvest losses. In the global food industry, natural is a powerful force as there is increasing resistance at regulatory and consumer levels against chemical food preservatives.

The results of the work showed that catfish (Clarias gariepinus) samples preserved with cold water, hot water and ethanolic extracts of bitter leaf differed from one another as well from the unpreserved sample in terms of the observed organoleptic characteristics. The results showed that unpreserved catfish can stay for 24 hours postslaughter before spoilage sets in. In the global food industry, natural is a powerful force as there is increasing resistance at regulatory and consumer levels against chemical food preservatives. Vernonia amygdalina has attracted attention to itself due to its possible antimicrobial activity. Vernonia amygdalina leaf extracts have been found active against a wide range of micro organisms that are of micro-biological importance for use in preservation. The shelf-life of catfish was extended by extracts of bitter leaf [Vernonia amygdalina] from $24 \mathrm{hrs}$ as seen in the unpreserved sample to $36 \mathrm{hrs}$ in the ethanolic extract preserved [Table 1]. It was also observed from the study that ethanol was the best method of extraction for the preservation of catfish [Clarias gariepinus] since it was found to extend the shelf life of fish to $36 \mathrm{hrs}$ compared to hot water than cold water extracts. Investigators in the past had also clearly shown that ethanolic extracts were more effective than hot and cold water extracts (Ibekwe et al, 2001, and Dutta 1993). It also corroborates the findings of Ephraim (2009) who used the cold water and hot water and ethanolic extracts of black pepper, seeds, grape peel and pawpaw seeds and found their ethanolic extracts most suitable for combating organisms that cause spoilage in fish. Hot water and cold water extracts of bitter leaf [Vernonia amygdalina] preserved fish for $20 \mathrm{hrs}$ (Table 2) before it was no longer fit for human consumption.

The inability of hot water to perform much is probably because some of the active ingredients like the flavonoids, saponins, tannin and glycosides in bitter leaf [Vernonia amygdalina] (Awe et al; 1999) are heat labile, while the reason for that of cold water 
TABLE 1: ORGANOLEPTIC CHARACTERISTICS OF UNPRESERVED CATFISH (Clarias gariepinus) MONITORED 4-HOURLY AT AMBIENT TEMPERATURE.

\begin{tabular}{|c|c|c|c|c|}
\hline Time(Hrs) & EYE & GILL & FLESH & SKIN \\
\hline 0 & Bright, iridescent appearance. & Bright Red, fresh odour. & Firm, smooth surface & Bright grey. \\
\hline 4 & Bright, iridescent cornea & Bright Red, fresh odour & Firm, smooth surface & $\begin{array}{c}\text { Dark grey at dorsal region, silvery at } \\
\text { ventral region, }\end{array}$ \\
\hline 8 & Bright, Iridescent cornea & Bright Red, fresh odour & Less firm, elastic & $\begin{array}{l}\text { Dark grey at dorsal region, silvery at } \\
\text { ventral region. }\end{array}$ \\
\hline 12 & Dark pupil & Pale Red, Neutral odour & Less firm, elastic & $\begin{array}{c}\text { Dark grey at dorsal region, whitish at } \\
\text { ventral region. }\end{array}$ \\
\hline 16 & Slightly convex, dark pupil & Pale Red, Neutral odour & Tough/ Slightly elastic & $\begin{array}{l}\text { Dark grey at dorsal region, whitish/ } \\
\text { creamy at ventral region. }\end{array}$ \\
\hline 20 & Flat, white pupil, & Pinkish, Neutral odour & Soft slightly elastic & $\begin{array}{l}\text { Dark grey and reddish at dorsal } \\
\text { region, creamy at ventral region. }\end{array}$ \\
\hline 24 & Flat, cloudy & Pinkish, Offensive odour & $\begin{array}{l}\text { Soft, red colour along } \\
\text { the column, slightly } \\
\text { elastic }\end{array}$ & $\begin{array}{l}\text { Dark grey and reddish at dorsal } \\
\text { region, creamy at ventral region. }\end{array}$ \\
\hline 28 & Flat, cloudy & $\begin{array}{l}\text { Bleached, slightly rancid/ } \\
\text { sour }\end{array}$ & $\begin{array}{l}\text { Very soft, not elastic } \\
\text { leaves finger indenta- } \\
\text { tions. }\end{array}$ & $\begin{array}{l}\text { Dark and reddish at dorsal region, } \\
\text { creamy and greenish at ventral } \\
\text { region. }\end{array}$ \\
\hline 32 & Flat, cloudy & $\begin{array}{l}\text { Bleached, slightly pun- } \\
\text { gent }\end{array}$ & $\begin{array}{l}\text { Very soft, tearing apart, } \\
\text { not elastic }\end{array}$ & $\begin{array}{l}\text { Dark and reddish at dorsal region, } \\
\text { creamy and greenish at ventral } \\
\text { region. }\end{array}$ \\
\hline 36 & Flat, cloudy & Bleached, offensive odour & $\begin{array}{c}\text { Very soft, tearing apart, } \\
\text { no elastic leaves finger } \\
\text { indentations. }\end{array}$ & $\begin{array}{l}\text { Dark, reddish and slightly greenish at } \\
\text { dorsal region, creamy and greenish at } \\
\text { ventral region. }\end{array}$ \\
\hline 40 & Flat, cloudy & Greyish, offensive odour & $\begin{array}{l}\text { Very soft, leaves finger } \\
\text { indentation, not elastic }\end{array}$ & $\begin{array}{l}\text { Dark, reddish and slightly greenish at } \\
\text { dorsal region, creamy and greenish at } \\
\text { ventral region }\end{array}$ \\
\hline 44 & Flat, cloudy & Greyish, offensive odour & $\begin{array}{l}\text { Very soft, leaves finger } \\
\text { indentation, not elastic, }\end{array}$ & $\begin{array}{c}\text { Dark, reddish and slightly greenish at } \\
\text { dorsal region, creamy and greenish at } \\
\text { ventral region }\end{array}$ \\
\hline 48 & Flat, cloudy & $\begin{array}{l}\text { Dull brown, offensive } \\
\text { odour }\end{array}$ & $\begin{array}{l}\text { Very soft, leaves finger } \\
\text { indentation, not elastic }\end{array}$ & $\begin{array}{l}\text { Dark, reddish and slightly greenish at } \\
\text { dorsal region, creamy and greenish at } \\
\text { ventral region }\end{array}$ \\
\hline
\end{tabular}


TABLE 2: ORGANOLEPTIC CHARACTERISTICS OF CATFISH (Clarias gariepinus) PRESERVED WITH ETHANOLIC EXTRACT OF BITTER LEAF

\begin{tabular}{|c|c|c|c|c|}
\hline Time(Hrs) & EYE & GILL & FLESH & SKIN \\
\hline 0 & Bright, convex, iridescent & Bright Red fresh odour. & Firm & Bright grey \\
\hline 4 & Bright, convex, iridescent & Bright Red fresh odour & Firm & Bright grey \\
\hline 8 & Convex, dark pupil & Pale red, fresh odour & Less firm, elastic & $\begin{array}{r}\text { Dark grey at dorsal region, silvery at } \\
\text { ventral region. }\end{array}$ \\
\hline 12 & Flat dark pupil & Pale red, Neutral odour & Less firm, elastic & $\begin{array}{r}\text { Dark grey at dorsal region, silvery at } \\
\text { ventral region. }\end{array}$ \\
\hline 16 & Slightly cloudy & $\begin{array}{r}\text { Brownish colour, neutral } \\
\text { odour }\end{array}$ & Less firm, slightly elastic & $\begin{array}{r}\text { Pale grey at dorsal region, silvery at } \\
\text { ventral region. }\end{array}$ \\
\hline 20 & Slightly cloudy and sunken & $\begin{array}{r}\text { Brownish colour, neutral } \\
\text { odour }\end{array}$ & Less firm slightly elastic & $\begin{array}{r}\begin{array}{r}\text { Pale grey at dorsal region, silvery at } \\
\text { ventral region. }\end{array} \\
\end{array}$ \\
\hline 24 & $\begin{array}{r}\text { Concave or sunken, slightly } \\
\text { cloudy }\end{array}$ & $\begin{array}{r}\text { Brownish colour, neutral } \\
\text { odour }\end{array}$ & Less firm, slightly elastic & $\begin{array}{r}\text { Pale grey and reddish at dorsal } \\
\text { region, slightly greenish at ventral } \\
\text { region. }\end{array}$ \\
\hline 28 & Sunken & $\begin{array}{r}\text { Brownish colour, neutral } \\
\text { odour }\end{array}$ & Less firm, slightly elastic & $\begin{array}{r}\text { Pale grey and reddish at dorsal } \\
\text { region, slightly creamy at ventral } \\
\text { region. }\end{array}$ \\
\hline 32 & Opaque pupil & $\begin{array}{r}\text { Brownish colour, neutral } \\
\text { odour }\end{array}$ & soft, slightly elastic & $\begin{array}{l}\text { Pale grey and reddish at dorsal re- } \\
\text { gion, creamy at ventral region. }\end{array}$ \\
\hline 36 & Concave, opaque pupil & $\begin{array}{r}\text { Brownish colour, slightly } \\
\text { pungent odour }\end{array}$ & $\begin{array}{r}\text { Very soft, leaves finger } \\
\text { indentations. }\end{array}$ & $\begin{array}{r}\text { Pale grey and reddish at dorsal } \\
\text { region, slightly greenish at ventral } \\
\text { region. }\end{array}$ \\
\hline 40 & Concave or sunken & $\begin{array}{r}\text { Brownish colour, pun- } \\
\text { gent odour }\end{array}$ & $\begin{array}{r}\text { Very soft, leaves finger } \\
\text { indentations. }\end{array}$ & $\begin{array}{r}\text { Dark and reddish at dorsal region, } \\
\text { greenish at ventral region. }\end{array}$ \\
\hline 44 & Opaque or Concave & $\begin{array}{r}\text { Brownish colour, pun- } \\
\text { gent odour }\end{array}$ & $\begin{array}{r}\text { Very soft, leaves finger } \\
\text { indentations. }\end{array}$ & $\begin{array}{r}\text { Dark and reddish at dorsal region, } \\
\text { greenish at ventral region. }\end{array}$ \\
\hline 48 & Concave or Sunken & $\begin{array}{r}\text { Brownish colour, pun- } \\
\text { gent odour }\end{array}$ & $\begin{array}{l}\text { Very soft, leaves finger } \\
\text { indentations. }\end{array}$ & $\begin{array}{l}\text { Dark and reddish at dorsal region, } \\
\text { greenish at ventral region. }\end{array}$ \\
\hline
\end{tabular}


TABLE 3: ORGANOLEPTIC CHARACTERISTICS OF CATFISH (Clarias gariepinus) PRESERVED WITH HOT WATER EXTRACT OF BITTER LEAF

\begin{tabular}{|c|c|c|c|c|}
\hline Time(Hrs) & EYE & GILL & FLESH & SKIN \\
\hline 0 & Bright, iridescent appearance. & $\begin{array}{r}\text { Bright Red, fresh } \\
\text { odour. }\end{array}$ & Firm & Bright grey \\
\hline 4 & Bright, convex, iridescent dark pupil & $\begin{array}{r}\text { Bright Red fresh } \\
\text { odour }\end{array}$ & Firm & $\begin{array}{r}\text { Dark grey at dorsal region, silvery at } \\
\text { ventral region., }\end{array}$ \\
\hline 8 & Convex, dark pupil & $\begin{array}{r}\text { Pale Red Neutral } \\
\text { odour }\end{array}$ & $\begin{array}{r}\text { Less firm, slightly } \\
\text { elastic }\end{array}$ & $\begin{array}{r}\text { Dark grey at dorsal region, silvery at } \\
\text { ventral region. }\end{array}$ \\
\hline 12 & Slightly convex, dark pupil & $\begin{array}{r}\text { Pale Red Neutral } \\
\text { odour }\end{array}$ & Less firm, slightly elastic & $\begin{array}{r}\text { Dark grey at dorsal region, silvery at } \\
\text { ventral region. }\end{array}$ \\
\hline 16 & Convex, dark pupil & $\begin{array}{r}\text { Pale Red Neutral } \\
\text { odour }\end{array}$ & Less firm, slightly elastic & $\begin{array}{r}\text { Dark grey at dorsal region, silvery at } \\
\text { ventral region. }\end{array}$ \\
\hline 20 & Flat, dark pupil, & $\begin{array}{r}\text { Pale Red slight } \\
\text { rancid } \\
\end{array}$ & Soft & $\begin{array}{r}\text { Dark grey at dorsal region, silvery at } \\
\text { ventral region. }\end{array}$ \\
\hline 24 & Flat, cloudy & $\begin{array}{r}\text { Pale Red offensive } \\
\text { odour }\end{array}$ & $\begin{array}{r}\text { Very soft, leaves finger } \\
\text { indentation }\end{array}$ & $\begin{array}{r}\text { Dark grey at dorsal region, silvery at } \\
\text { ventral region. }\end{array}$ \\
\hline 28 & Flat, cloudy & $\begin{array}{r}\text { Brownish colour, } \\
\text { offensive odour }\end{array}$ & $\begin{array}{r}\text { Very soft, leaves finger- } \\
\text { print } \\
\text { indentation }\end{array}$ & $\begin{array}{r}\text { Dark grey at dorsal region, silvery at } \\
\text { ventral region. }\end{array}$ \\
\hline 32 & Flat, cloudy & $\begin{array}{l}\text { Brownish colour, } \\
\text { offensive odour }\end{array}$ & $\begin{array}{r}\text { Very soft, leaves finger- } \\
\text { print } \\
\text { Indentation, not elastic }\end{array}$ & $\begin{array}{r}\text { Dark grey at dorsal region, silvery at } \\
\text { ventral region. }\end{array}$ \\
\hline 36 & Flat, cloudy & $\begin{array}{l}\text { Brownish colour, } \\
\text { offensive odour }\end{array}$ & $\begin{array}{r}\text { Very soft, leaves finger } \\
\text { indentation }\end{array}$ & $\begin{array}{r}\text { Red colour at vertebral column. } \\
\text { Dark grey at dorsal region, silvery at } \\
\text { ventral region }\end{array}$ \\
\hline 40 & Flat, cloudy & $\begin{array}{l}\text { Brownish colour, } \\
\text { offensive odour }\end{array}$ & $\begin{array}{r}\text { Very soft, leaves finger } \\
\text { indentation }\end{array}$ & $\begin{array}{r}\text { Dark grey at dorsal region, silvery at } \\
\text { ventral region. } \\
\text { Red colour at vertebral column }\end{array}$ \\
\hline 44 & Slightly convex & $\begin{array}{l}\text { Brownish colour, } \\
\text { offensive odour }\end{array}$ & $\begin{array}{r}\text { Very soft, leaves finger } \\
\text { indentation }\end{array}$ & $\begin{array}{r}\text { Dark grey at dorsal region, silvery at } \\
\text { ventral region. } \\
\text { Red colour at vertebral column }\end{array}$ \\
\hline 48 & Concave on sunken & $\begin{array}{l}\text { Brownish colour, } \\
\text { offensive odour }\end{array}$ & $\begin{array}{r}\text { Very soft, leaves finger } \\
\text { indentation }\end{array}$ & $\begin{array}{r}\text { Dark grey at dorsal region, silvery at } \\
\text { ventral region. } \\
\text { Red colour at vertebral column. }\end{array}$ \\
\hline
\end{tabular}


TABLE 4: ORGANOLEPTIC CHARACTERISTICS OF CATFISH (Clarias gariepinus) PRESERVED WITH COLD WATER EXTRACT OF BITTER LEAF (Vernonia amgdalina )

\begin{tabular}{|c|c|c|c|c|}
\hline Time(Hrs) & EYE & GILL & FLESH & SKIN \\
\hline 0 & $\begin{array}{r}\text { Bright, convex, iridescent Dark pupil } \\
\text { cornea }\end{array}$ & $\begin{array}{r}\text { Bright red, fresh } \\
\text { odour. }\end{array}$ & Firm & Bright, grey at dorsal region \\
\hline 4 & convex, iridescent dark pupil & $\begin{array}{r}\text { pale red, neutral } \\
\text { odour }\end{array}$ & Firm, & $\begin{array}{r}\text { Bright grey at dorsal region, silvery at } \\
\text { ventral region., }\end{array}$ \\
\hline 8 & Convex, dark pupil & $\begin{array}{r}\text { pale red, neutral } \\
\text { odour }\end{array}$ & $\begin{array}{r}\text { Less firm, slightly } \\
\text { elastic }\end{array}$ & $\begin{array}{r}\text { Dark grey at dorsal region, silvery at } \\
\text { ventral region. }\end{array}$ \\
\hline 12 & Convex loss of brightness & $\begin{array}{r}\text { pale red, neutral } \\
\text { odour }\end{array}$ & $\begin{array}{r}\text { Less firm, slightly } \\
\text { elastic }\end{array}$ & $\begin{array}{r}\text { Dark grey at dorsal region, silvery at } \\
\text { ventral region. }\end{array}$ \\
\hline 16 & $\begin{array}{r}\text { Slightly convex, white pupil, grey } \\
\text { cornea }\end{array}$ & $\begin{array}{r}\text { pale red, neutral } \\
\text { odour }\end{array}$ & $\begin{array}{r}\text { Less firm slightly } \\
\text { elastic }\end{array}$ & $\begin{array}{r}\text { Dark grey at dorsal region, silvery at } \\
\text { ventral region. }\end{array}$ \\
\hline 20 & Flat, cloudy & $\begin{array}{r}\text { pale red, neutral } \\
\text { odour }\end{array}$ & Soft & $\begin{array}{r}\text { Dark grey at dorsal region, silvery at } \\
\text { ventral region. }\end{array}$ \\
\hline 24 & Flat, cloudy, loss of brightness & $\begin{array}{r}\text { pale red, } \\
\text { Offensive Odour }\end{array}$ & Soft & $\begin{array}{r}\text { Dark grey at dorsal region, silvery at } \\
\text { ventral region. }\end{array}$ \\
\hline 28 & Flat, dull & $\begin{array}{r}\text { pale red, offensive } \\
\text { odour }\end{array}$ & Tough, leathery & $\begin{array}{r}\text { Dark grey at dorsal region, silvery at } \\
\text { ventral region. }\end{array}$ \\
\hline 32 & Flat, loss of brightness & $\begin{array}{r}\text { Pinkish, offensive } \\
\text { odour }\end{array}$ & $\begin{array}{r}\text { Very Soft, leaves finger } \\
\text { indentation }\end{array}$ & Dark red at the vertebral column \\
\hline 36 & Flat, cloudy loss of brightness & $\begin{array}{l}\text { Whitish in colour, } \\
\text { offensive odour }\end{array}$ & $\begin{array}{r}\text { Very soft, leaves finger } \\
\text { indentation }\end{array}$ & Dull, dark, red, \\
\hline 40 & Flat, cloudy & $\begin{array}{r}\text { Bleached, offensive } \\
\text { odour }\end{array}$ & $\begin{array}{l}\text { Very soft, leaves finger } \\
\text { indentation not elastic. }\end{array}$ & Slime \\
\hline 44 & Concave & $\begin{array}{r}\text { Bleached, offensive } \\
\text { odour }\end{array}$ & $\begin{array}{l}\text { Very soft, leaves finger } \\
\text { indentation, not elastic }\end{array}$ & $\begin{array}{r}\text { Dark grey at dorsal region, creamy at } \\
\text { ventral region. }\end{array}$ \\
\hline 48 & Concave or Sunken & $\begin{array}{r}\text { Bleached, offensive } \\
\text { odour }\end{array}$ & $\begin{array}{l}\text { Very soft, leaves finger } \\
\text { indentation, not elastic }\end{array}$ & $\begin{array}{l}\text { Dark grey at dorsal region. } \\
\text { Creamy at ventral region. }\end{array}$ \\
\hline
\end{tabular}


could be that these compounds cannot be easily liberated by it. It can thus be deduced that hot water and coldwater as solvents of extraction for bitter leaf is less effective than the ethanolic extract. The unpreserved sample remained fit for consumption for $24 \mathrm{hrs}$ before spoilage could set in. This is in line with the findings of Ephraim (2009) who monitored catfish [Clarias gariepinus] at an ambient temperature but differed from that of Ajibola (2010) and Olateju (2010) as the unpreserved fish sample from these studies stayed till the 20th hour. The reason for this study may be attributed to the ambient and environmental temperatures as at the time of experiment. The study carried out by Ephraim (2009) was in Abeokuta Ogun State showed a variation in environmental while that of Ajibola (2010) and Olateju (2010) might have resulted in a different ambient temperature as at the time of experiment. This work confirms the efficacy of ethanolic extract of bitter leaf (Vernonia amygdalina) and its potential as organic preservatives in fisheries and aquaculture to reduce post-harvest losses.

\section{Conclusion}

It can thus be concluded from this study that ethanolic extract of Vernonia amygdalina [bitter leaf] is best for preserving catfish [Clarias gariepinus] in order to extend its shelf life. Bitter leaf is able to do this because of its preservative ability which stems from the phytochemical compounds present in it. These compounds where also discovered from this work to be heat labile. It is a yet to be exploited natural preservative for catfish through which post harvest losses in fisheries can be reduced.

\section{References}

1. Ajibola O.J (2010) Ethanolic Extract of black pepper seed as a preservative for processed Fresh catfish (Clarias gariepinus). ND project, FCFFT New Bussa, Niger State. Pp 18-19.

2. Awe, S.O; Makinde, J.M and Olajide, O.A (1999) Cathartic effect of the leaf extract of Vernonia amygdalina Fitoterapia
3. Azu, N.C. and Onyeagba, R. A. (2007) Antimicrobial Properties of extracts Allium cepa (onions) and Zingiber officinale (ginger) on Escherichia coli, Salmonella typhi and Bacillus subtilis. The Internet. Journal of tropical Medicine. 3(2).1-3p

4. Bensi, M.L.K; Osuji, P.O; Tuah, A.K and Umunna, N.N (1995) Vernonia amygdalina as a supplement to Teff straw (Eragrostis tef) fed to Ethiopian menz Sheep. Agroforestry systems 31 (3). Pp. 229-241.

5. Butter, G.W and Bailey, R.W (1973) Chemistry and Biochemistry of Herbage. Vol.1 Academic press, London and New York.

6. Clucas, I.J (Compiler) (1985) Fish handling, Preservation and Processing in the tropics. Part 1, Tropical Development and research institute London $141 \mathrm{pp}$.

7. Dutta, A.C (1993) Botany for degree students. 5th edition. Oxford University Press, Oxford pp. 810-844.

8. Ephraim, R.N (2009) Anti microbial properties of extracts of Carica papaya, Citrus paradisa and Pepper guineese on micro organism associated with Catfish [Clarias gariepinus] spoilage. MAF Project, University of Agriculture, Abeokuta, Ogun State. Pp1-36

9. Ibekwe, V.I; Nnanyere N.F and Akujobi C.O (2001) Studies on anti bacterial Activity and phytochemical qualities of extracts of orange peels. Int. J. Environ; Health and Human Development 2(1):41-46

10. Ikeme, A.I. and Bhandary, C.S. (2001). Effect of spice treatment on the quality of hot-smoked mackerel (Scomber scombus) presented at the seventh FAO Expert Consultation in Africa, Senegal. 1-10pp.

11. Olateju O.P (2010) Shelf life of grape fruit peel preserved fresh catfish (Clarias gariepinus). ND Project, FCFFT New Bussa, Niger State. Pp 17-18. 